\title{
THE EFFECT OF CONTINUOUS POSITIVE AIRWAY PRESSURE ON GLUCOSE EXCURSIONS IN DIABETICS WITH SLEEP-DISORDERED BREATHING: THE RESULTS OF CONTINUOUS GLUCOSE MONITORING
}

\author{
M. Pallayova*, V. Donic*, v. Donicova*, Z. Tomori* and S. Gresova* \\ *Dept of Physiology and Sleep Laboratory, Faculty of Medicine, Pavol Jozef Safarik University, Kosice, Slovakia \\ \#Dept of Internal Medicine and Diabetology, Outpatient Clinic, Kosice, Slovakia
}

WINNING ABSTRACT: Sleep-disordered breathing (SDB) is often associated with impaired glucose metabolism. The study aimed at assessing immediate effect of CPAP on glucose excursions in type 2 diabetic patients with SDB measured with 72hour continuous glucose monitoring system (CGMS).

8 type 2 diabetic patients with SDB (men, age 48,13 $\pm 4,91$ years, BMI 34,06 $\pm 7,41 \mathrm{~kg} \cdot \mathrm{m}^{-2}$, HbA1c 7,3 $\pm 1,4 \%$ ) underwent 2 overnight polysomnographic examinations including diagnostic night and CPAP night. CGMS was applied on both occasions. Statistical analyses included paired Student's t-test.

CPAP decreased apnoea-hypopnoea index (AHI) from $57,64 \pm 9,64 \cdot h^{-1}$ to $8,05 \pm 4,42 \cdot h^{-1}$ (p $\left.<0,0001\right)$ with significant improvement of saturation. Frequent episodes of sleep apnoea/hypopnoea and severe oxygen desaturation were followed by significant rise in blood glucose of up to $12,3 \mathrm{mmol} \cdot \mathrm{I}^{-1}$. Duration of post-hypoxic hyperglycemia was $50 \pm 10,79 \mathrm{~min}$ and its climax tended to be appeared up to $45 \mathrm{~min}$ post-hypoxia. Nocturnal hyperglycemia strongly correlated with severe oxygen desaturation. Nocturnal glucose values were significantly higher during diagnostic night than during CPAP night $\left(8,19 \pm 0,99 \mathrm{mmol} \cdot I^{-1}\right.$ versus $\left.6,77 \pm 1,47 \mathrm{mmol} \cdot \mathrm{I}^{-1} ; \mathrm{p}<0,0001\right)$. CGMS also showed improved preprandial and 1,5 -hour postprandial glucose levels for breakfast after CPAP night. The improvement in overall glucose levels was much greater in patients with $\mathrm{BMI}<\mathbf{3 0} \mathrm{kg} \cdot \mathrm{m}^{-2}$ than in more obese patients.

The results suggest that nocturnal hyperglycemia is closely related to desaturation and CPAP treatment may have an immediate decreasing effect on blood glucose in type 2 diabetic patients with SDB.

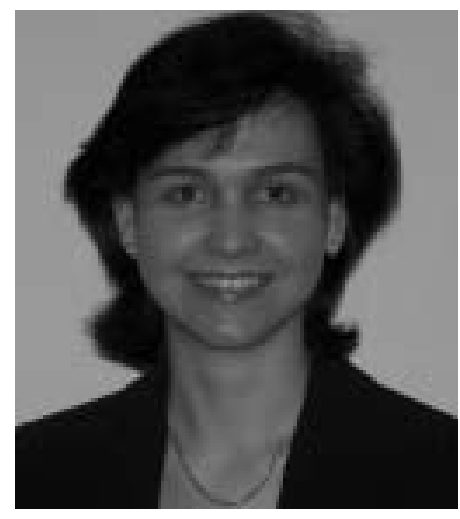

Maria Pallayova

Dept of Physiology and Sleep Laboratory, Faculty of

Medicine, PJ Safarik University, Kosice, Slovakia

\section{MY JOB AND THE UNIT IN WHICH I WORK}

My current position is medical doctor, lecturer and PhD fellow at the Dept of Physiology and Sleep Laboratory in the Faculty of Medicine, PJ Safarik University (Kosice, Slovakia). Our department is a pre-clinical department of a medical school situated adjacent to the Luis Pasteur University Hospital in Kosice. The research at the department covers the complex study of upper airway reflexes with clinical-physiological implications of the gasp-like aspiration reflex and autoresuscitation by gasping analysed in animals. Results of research on upper airway reflexes have been published in $>300$ papers, including four monographs and abstracts, with
$>640$ citations to these publications, according to the Science Citation Index [1-3]. Another large research area at the department is devoted to sleep medicine in humans, with diagnosis, therapy and prevention of sleep-related breathing disorders and their cardiovascular, neurobehavioural, metabolic and endocrine consequences. Our team participates in COST Action B26 (European Cooperation in the field of Scientific and Technical Research), which aims to establish a permanent network among outstanding groups actively contributing to studies on obstructive sleep apnoea/hypopnoea syndrome (OSAHS) in different European countries, in order to pursue advances in clinical and basic research on OSAHS and cardiovascular risk. Diagnosis and treatment of sleeprelated breathing disorders is pursued in a specialised sleep laboratory. During the diagnostic night each patient is investigated using overnight polysomnography (PSG) consisting of continuous polygraphical recordings for an entire night with standardised equipment (Alice 3; Healthdyne Technologies, Marietta, GA, USA). It is also possible to measure blood pressure (Cardiotens 1.34; Meditech, Budapest, Hungary) and glucose levels via a continuous glucose monitoring system (CGMS; Medtronic MiniMed, Bratislava, Slovakia) continuously in parallel with PSG. Patients with severe sleep-disordered breathing (SDB) are usually treated with continuous positive airway pressure (CPAP).

Besides my clinical research work, I also participate in the practical teaching of undergraduate students of general medicine, who are in the second year of their 6 yr-long course. There are two semesters of human physiology, which is an important science studying the way that cells, tissues and organs work in people. 


\section{MY WINNING ABSTRACT AS PART OF MY RESEARCH}

Starting as early as my undergraduate medical studies (19992005), I have conducted several preliminary studies using CGMS in children and adult diabetic patients in Slovakia. I participated in several students' scientific conferences with a total of five of my own research works, including Best Paper Awards, and first and third prizes. I started my $\mathrm{PhD}$ in September 2005. My thesis "Continuous glucose monitoring in patients with diabetes and sleep-related breathing disorders" is aimed at metabolic and endocrine aspects of SDB. I perform CGMS in parallel with overnight PSG in diabetics in the sleep laboratory to determine the prevalence, cause and extent of nocturnal glycaemic excursions. The aim of my research work is to evaluate patients' nocturnal glycaemic responses to sleep, sleep apnoea/hypopnoea, severe oxygen desaturation, arousal and CPAP therapy in order to find more associations between sleep apnoea and glucose control with potential therapeutic implications. Performance of CGMS in parallel with overnight PSG in the sleep laboratory allows us to also reveal unrecognised nocturnal glucose patterns, including dangerous unknown post-apnoeic hyperglycaemia previously unseen using other methods. Every small detail during the night can now be monitored, and an impact of sleep apnoea and an immediate CPAP effect on glycaemia can also be assessed. CGMS technology has been shown to be an important advance in management of intensified insulin-treated diabetic patients [4] and also in type 2 diabetics with SDB (unpublished data, M. Pallayova).

\section{MY RESEARCH AS PART OF MY WORKING GROUP/ RESEARCH TEAM}

I am part of a team lead by the Head of the Department, Prof. Viliam Donic who has also been my supervisor and a tremendous role model for me as a scientist and physician. I must also acknowledge and thank Prof. Zoltan Tomori, and all my colleagues, collaborators and the rest of our team for their contribution to medicine, their ideas and their hard work. Our team has been involved in many research projects. The most important activities performed during our former participation in an European project, TEMPUS, include: the establishment of three sleep laboratories at three medical schools in Slovakia; the introduction of an interdisciplinary subject, "Sleep Medicine", into undergraduate medical study in Slovakia; the organisation of 20 short visits of teachers to partner universities in Antwerp (Belgium), Dublin (Ireland) and Strasbourg (France) to study sleep medicine; the publication of a monograph, Basics of Sleep Medicine [5], to support undergraduate and postgraduate education; overnight PSG examination of $>600$ patients with SDB and the introduction of CPAP therapy in Slovakia; and a complex study of cardiovascular effects of OSAHS in an international project (COST Action B26) comprising 14 European countries. The main topics of a currently running APVV (Slovak Research and Development Agency) research project include: the role of reflexes from upper airways in evoking various types of arousal in sleep apnoea and other patients; aspiration reflex as a model for interruption of severe paroxysmal dysfunctions in animal experiments and in humans; running a sleep laboratory with complex analysis of cardiorespiratory, neurobehavioural, metabolic and endocrine effects of sleep apnoea, their diagnosis, therapy and prevention. My research fits well into the sleep medicine research area of our department. I focus my research specifically on endocrine and metabolic aspects of sleep apnoea. Similar clustering of cardiovascular disease risk factors, one of which involves insulin resistance characteristic in type 2 diabetes, is also typical for patients with SDB. Sleep apnoea goes with diabetes and diabetes goes with sleep apnoea, and together these present a very dangerous combination. Diabetes itself has been described as a silent killer because of its late complications affecting the eyes, kidneys, nerves and large and small blood vessels, being strongly related to hyperglycaemias. The goal of effective diabetes management is to control blood glucose levels by keeping them within a target range, which is the best way to minimise the risk of these complications. Untreated sleep apnoea with recurrent episodes of airflow obstruction and hypoxaemia may adversely influence metabolic and glycaemic control, and contributes to the development of late diabetes complications. Several studies have suggested that OSAHS is pathophysiologically related to impaired glucose homeostasis and that CPAP is an important therapy with the potential to also improve insulin responsiveness. However, current data regarding the effects of CPAP on glycaemia are conflicting and often lacking in proper investigational methods. We hypothesise that CPAP may improve glycaemic control in sleep apnoea patients who also suffer from type 2 diabetes. Our aim was to assess an immediate CPAP effect on glycaemia in patients with severe SDB and concomitant type 2 diabetes using the CGMS in parallel with overnight PSG, which is the subject of my abstract.

\section{THE IMPACT OF MY WORK ON CLINICAL OR RESEARCH PRACTICE}

Obstructive sleep apnoea is not only an obstruction, it is a cardiovascular and metabolic nightmare. SDB is widely unrecognised in the type 2 diabetic population and this condition is linked to increased use of medical resources. The diagnosis and treatment of SDB is a growing and vitally important field. CGMS in parallel with overnight PSG has a potential to also detect abnormal nocturnal glucose patterns related to OSAHS not previously identified. Our results indicate that nocturnal increment in blood glucose expressed as the hyperglycaemic area under the glucose concentration curve during 8-h period of night was significantly higher for diagnostic nights (before CPAP) compared with CPAP nights, and was strongly negatively correlated with severe oxygen desaturation. Mean nocturnal glucose values were significantly higher during diagnostic nights as compared with CPAP nights. CGMS has also shown improved pre- and postprandial glucose levels for breakfast after the CPAP night. We suggest that improved glucose control under CPAP may reflect decreased sympathetic activity and improved insulin sensitivity, which is determined not only by obesity but by sleep apnoea. Our results indicate that CPAP is a highly effective tool for restoring normal breathing in OSAHS and has the potential to improve glycaemic control in patients with concomitant type 2 diabetes. Diagnosis and treatment of sleep apnoea can contribute to an improved quality of life and may decrease apnoea- and also diabetes-related morbidity and mortality. Detailed data from CGMS and parallel PSG can assist in designing an individualised diabetes and sleep 
apnoea treatment programme and may facilitate comprehensive management recommendations.

\section{REFERENCES}

1 Tomori Z, Widdicombe JG. Muscular, bronchomotor and cardiovascular reflexes elicited by mechanical stimulation of the respiratory tract. J Physiol 1969; 200: 25-49.

2 Korpas J, Tomori Z. Cough and Other Respiratory Reflexes. Karger, Basel, 1979; p. 356.
3 Jakus J, Tomori Z, Stransky A. Neuronal Determinants of Breathing, Coughing, and Related Motor Behaviours. Wist, Martin, 2004; p. 335.

4 Pallayova M, Donicova V, Donic V. Clinical utility of the continuous glucose monitoring system in intensified insulin treated patients. $5^{\text {th }}$ Annual Diabetes Technology Meeting Syllabus, San Francisco, USA, November 10-12, 2005; Abstract A102.

5 Tomori Z, Redhammer R, Donic V. Basics of Sleep Medicine. VLA SNP, Kosice, 1999; p. 368. 